\title{
HYBRID COLLECTORS USING THIN-FILM TECHNOLOGY
}

\author{
R. Platz*, D. Fischer, M.-A. Zufferey, J.A. Anna Selvan, A. Haller ${ }^{\ddagger}$ and A. Shah \\ Institut de Microtechnique, Université de Neuchâtel, Rue A.-L. Breguet 2, 2000 Neuchâtel, Switzerland \\ ${ }^{\ddagger}$ E. Schweizer AG, 8908 Hedingen, Switzerland \\ *present address: Department of Electrical Engineering, Princeton University, Princeton, NJ 08544 \\ E-Mail: rplatz@ee.princeton.edumailto:rplatz@ee.princeton.edu http://www.princeton.edu/ rplatz/
}

\begin{abstract}
Amorphous silicon (a-Si:H) based solar cells are highly interesting in the context of hybrid (i.e. photovoltaic/ thermal) solar energy conversion. First, their large area capability and the variety of possible substrate materials permit to apply a-Si:H PV modules directly on the surface of conventional heat collectors at low cost. Further, the low temperature coefficient of a-Si:H cells $(0.1 \% / \mathrm{K})$ allows operation of a-Si:H solar modules at temperatures as high as $100^{\circ} \mathrm{C}$ without substantial power loss. We focus on the thermal performance of such hybrid collectors based on a-Si:H cells, with emphasis on a $\mathrm{ZnO}$ coat on top of the solar cell. ZnO can be "tuned" to absorb the infrared part of the sunlight and, at the same time, its emission coefficient for heat-radiation is nearly as low as that of optimized selective surfaces used in thermal collectors. We propose a collector structure with a high potential for the thermal conversion efficiency while maintaining a high electrical conversion efficiency.
\end{abstract}

\section{Introduction}

The lower cost of amorphous silicon solar modules as compared to other silicon based techniques [1] as well as the possibility of deposition directly on large surfaces of different substrates [2-4] offer substantial advantages for this thin-film silicon technology. Furthermore, it has been shown that the temperature coefficient of amorphous silicon solar cells $(-0.1 \% / \mathrm{K}[5])$ is much smaller than that of crystalline silicon solar cells $(-0.4 \% / \mathrm{K}[5])$. This allows to operate a-Si:H modules at temperatures up to $100^{\circ} \mathrm{C}$ without substantial power losses. Consequently, the hybrid collector can be used to produce valuable medium to high temperature heat without compromising the PV output (this is definitely not the case for $\mathrm{C}-\mathrm{Si}$ based hybrid conversion). Recently, it has been shown, that the effective temperature coefficient of amorphous silicon solar cells may be even slightly positive, if one takes into account the strongly reduced degradation effect if cells are operated at higher temperatures $[6,7]$.

\section{Hybrid collectors}

A schematic crossection view of a typical hybrid collector is shown in Fig.1. The absorber surface of a conventional thermal collector is herein replaced by a solar module. This means that the optimized selective absorber surface with a strong absorption in the range of the sun spectrum and low radiation in the far-IR is replaced by a surface whose properties are basically given by the solar module and thereby in general not optimized for heat collector purposes.

The present paper deals with two aspects of optimizing the thermal efficiency of an a-Si:H based hybrid collector: Reduction of the absorption losses due to reflection of the IR part of the sun spectrum (term A in Fig.1.) and minimization of the thermal losses due to thermal radiation of the absorber surface in the far-IR (term B in Fig.1.).

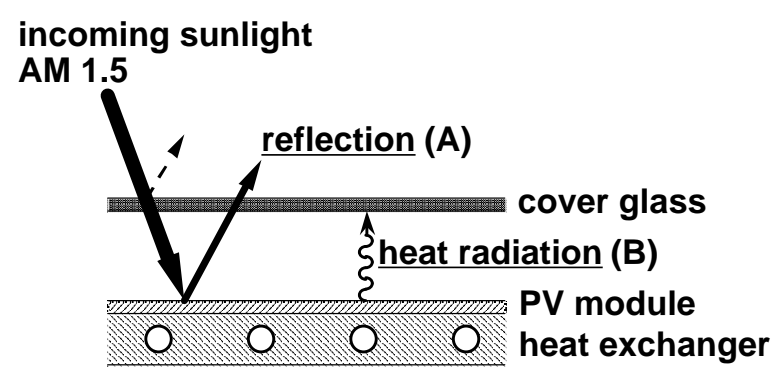

Fig.1.: Schematical view of a typical hybrid (thermal/ photovoltaic) solar module. The cover glass is optional, for high-performance collectors it is, however, generally applied as it reduces considerably heat losses and thereby increases the thermal efficiency of the module.

\section{Total sunlight absorption of commercial and laboratory solar cell structures - reflection losses}

Fig.2. shows the total reflection (measured by employing an integrating sphere in a VIS/UV spectrometer) for different commercial modules and a laboratory $p$-i-n cell. The total absorbed power under AM1.5 conditions for those samples is given in Table 1, employing $A=1-R$ (where $A$ is absorption and $R$ is reflection). Reflection is for all types of solar cells very low for wavelengths smaller than corresponding to the optical gap. There is a pronounced increase for higher wavelengths $(>700 \mathrm{~nm}$ for $\mathrm{a}-\mathrm{Si}: \mathrm{H}$ and $>1200 \mathrm{~nm}$ for $\mathrm{c}-\mathrm{Si}$ ). Namely in the IR region of the sun spectrum, reflection is much higher than for a conventional optimized absorber surface ( $X$, Fig.2.). Crystalline silicon based modules are quite well-suited for complete absorption of the sun spectrum, due to their antireflective coating on top of the cells and due to their low optical gap. However, we believe that for economic reasons, as stated in the introduction, as well as due to their poor behavior at high temperatures, crystalline silicon solar cells do not present a relevant alternative to a-Si:H solar cells in the field of hybrid collectors. 
Thus, one of the major loss terms in a-Si:H hybrid collectors turns out to be the reduced thermal efficiency due to an incomplete absorption of the light in the nearinfrared range in PV modules [8] (loss A in Fig.1.). 14-22\% of the indicent energy is reflected in the case of the investigated amorphous silicon cells.

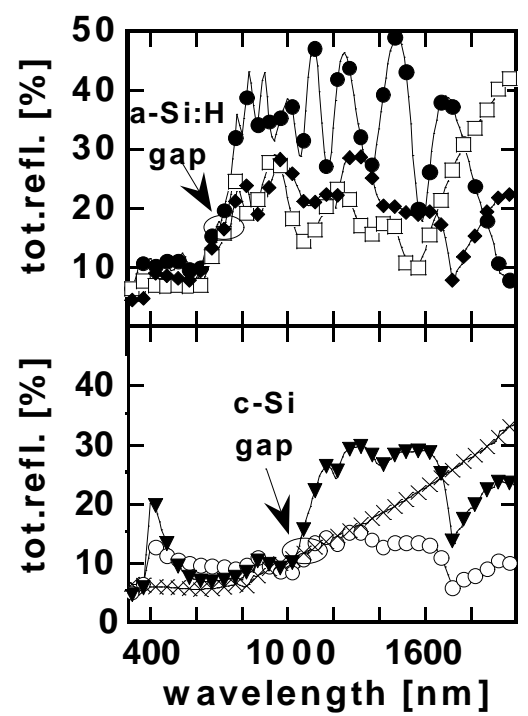

Fig.2.: Measured total reflection for different commercial and laboratory cell structures as a function of the wavelength in the range of the sun spectrum. Symbols are explained in Table 1.

\begin{tabular}{|l|l|l|}
\hline sample & $\begin{array}{l}\text { abs. P } \\
{\left[\mathrm{W} / \mathrm{m}^{2}\right]}\end{array}$ & $\begin{array}{l}\% \text { of } \\
\text { AM1.5 }\end{array}$ \\
\hline AM1.5 (not shown in Fig.1) & 957 & 100 \\
\hline 1) IMT p-i-n cell - & 744 & 78 \\
\hline 2) PST p-i-n-p-i-n cell $\square$ & 824 & 86 \\
\hline 3) Unisolar LM-603 $\bullet$ & 812 & 85 \\
\hline 4) Arco M-82 O (c-Si) & 858 & 90 \\
\hline $\begin{array}{l}\text { 5) Solarex MSX18L } \nabla \\
\text { (poly-Si) }\end{array}$ & 838 & 88 \\
\hline $\begin{array}{l}\text { 6) Commercial selective } \\
\text { absorber } \times\end{array}$ & 875 & 91 \\
\hline
\end{tabular}

Table 1.: Calculated absorbed power

$\left(=\int A M 1.5(1-R) d \lambda\right)$ for the samples of Fig.1. and percentage of total power in the AM1.5 spectrum. Power values are calculated by multiplying the absorption $(=1-R)$ with the sun spectrum and integrating over all wavelengths. Samples 1) and 2) have a glass surface on top, samples 3)-5) are encapsulated with a more or less textured plastic surface on top.

Therefore, in order to increase the thermal performance of a hybrid collector, the absorption of the IR part of the sun spectrum should be increased. This should be done without affecting the electrical properties of the module. In the following we will present a possible way to realize such an improvement.

\section{Tunable IR-absorption of $\mathrm{ZnO}$}

$\mathrm{ZnO}$ can be tailored w.r. to its optical and electrical properties. It is widely used in thin film solar cell technology and its process technology is well established. The near-infrared absorption properties of $\mathrm{ZnO}$ are found to depend on the deposition parameters; in fact, it is possible to deposit $\mathrm{ZnO}$ which is highly transparent in the visible and strongly absorbing in the near-infrared range of the sun spectrum due to free-carrier absorption [9]. Fig.3. shows transmission, absorption and reflection of such a $\mathrm{ZnO}$ film in the wavelength range of the sun spectrum. The transmission in the range of current generation in the amorphous cell $(350-700 \mathrm{~nm})$ is high. For wavelengths not absorbed in the a-Si: $\mathrm{H}$, the $\mathrm{ZnO}$ film becomes absorbing, converting the near-IR part of the sunlight into heat which can be collected in a hybrid module.

For even higher wavelengths, highly doped TCOs behave similarly to metals, showing a high IR reflectance due to free carriers. This behavior is basically the same for all common TCOs (see e.g. [10] for ITO) and makes those materials suited as so-called heat-mirrors, as will be discussed in the following section of this paper.

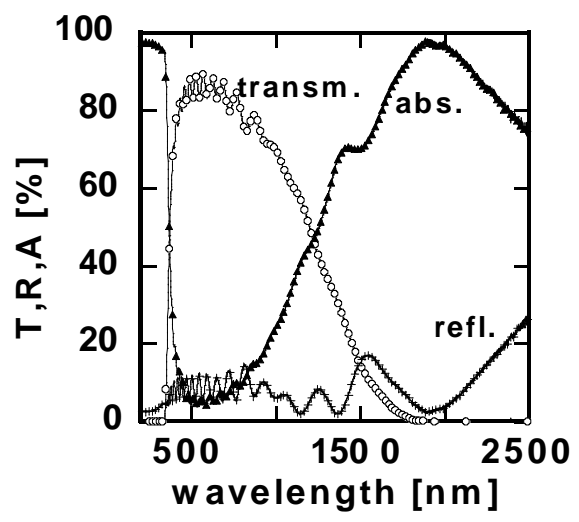

Fig.3.: Measured absorption, reflection and transmission data of a $1.33 \mu \mathrm{m}$ thick sputtered $\mathrm{ZnO}$ film on glass.

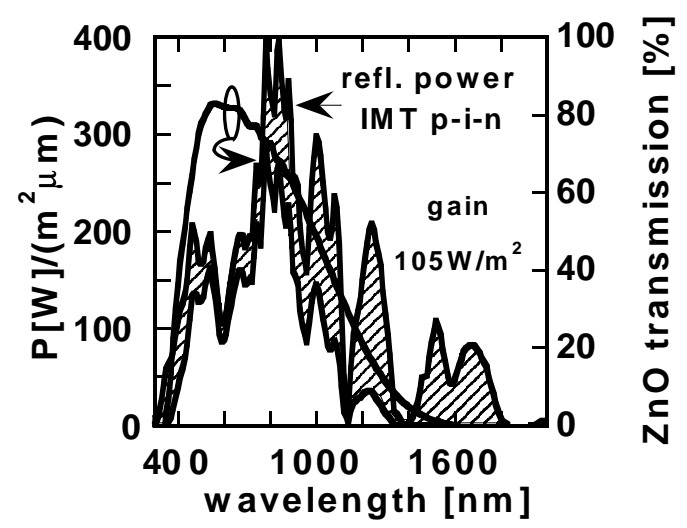

Fig.4.: Reflected power for the IMT sample (\#1 in Tab.1.) and calculated reflected power after and additional pass through a selectively absorbing $\mathrm{ZnO}$ layer.

In the case of the IMT p-i-n sample of Table 1, light corresponding to $213 \mathrm{~W} / \mathrm{m}^{2}$ is reflected, i.e. more than $20 \%$ of the total available energy. This value can be reduced by inserting a suited $\mathrm{ZnO}$ layer, as described above, in the path of the non absorbed IR light. Fig.4. shows a calculated example for the case of this $p-i-n$ sample using a $\mathrm{ZnO}$ absorption behavior as shown in 
Fig.3. The gain was calculated in a first approximation by multiplying the reflection spectra of the IMT p-i-n sample (weighted with the AM1.5 sun spectrum, see formula in the caption of table 1) with the $\mathrm{ZnO}$ transmission. About 100 $\mathrm{W} / \mathrm{m}^{2}$ could be additionally absorbed in this case.

In order to confirm this concept experimentally, we deposited a 400nm thick $p$-i-n cell on an Asahi U-type TCO substrate. One piece of the sample was contacted using a conventional ITO/Ag back contact, the other piece was contacted using $\mathrm{ZnO} / \mathrm{Ag}$. The silver deposition was done in the same run for both samples. The deposition of the $\mathrm{ZnO}$ layer was tuned in order to yield the desired IR absorption properties as described above.

Fig.5. shows the measured reflection curves of both samples weighted with the AM1.5 sun spectrum. There is a clearly reduced reflection of the $\mathrm{ZnO} / \mathrm{Ag}$ sample for red and IR wavelengths. Integration of the reflection spectra of Fig.5. yields for the ITO/Ag sample an absorbed power density of $775 \mathrm{~W} / \mathrm{m}^{2}$, for the $\mathrm{ZnO} / \mathrm{Ag}$ sample this value is of $808 \mathrm{~W} / \mathrm{m}^{2}$ which means a gain of $33 \mathrm{~W} / \mathrm{m}^{2}$. Note that this gain can be achieved without affecting the electrical properties of the cell, namely without reducing the current generated in the cell. A cell with such a $\mathrm{ZnO} / \mathrm{Ag}$ back contact as described above yielded $9 \%$ efficiency $\left(\mathrm{V}=0.86 \mathrm{~V}, \mathrm{FF}=69 \%, \mathrm{I}=15.2 \mathrm{~mA} / \mathrm{cm}^{2}\right)$, the same cell with an ITO/Ag back contact yielded an efficiency of $9.7 \%$ $\left(V_{o c}=0.86 \mathrm{~V}, F F=74 \%, I=15.2 \mathrm{~mA} / \mathrm{cm}^{2}\right)$. The lower $F F$ for the $\mathrm{ZnO}$ cell is mainly due to experimental problems aligning $\mathrm{ZnO}$ and $\mathrm{Ag}$ depositon through a mask as both depositions are made in different systems for this laboratory sample. This results in lateral collection with an incresed series resistance which reduces the fill factor.

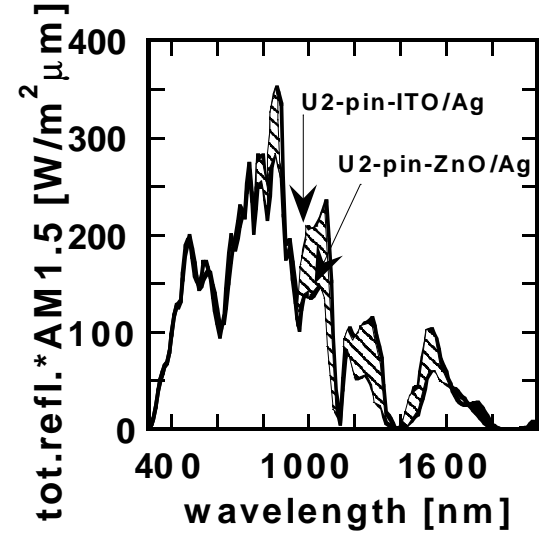

Fig.5.: Comparison of reflection measurements for samples of amorphous silicon on TCO (Asahi U-type) with an ITO/Ag and a $\mathrm{ZnO} / \mathrm{Ag}$ back contact (otherwise identical). The gain in IR absorption by the $\mathrm{ZnO}$ layer corresponds to $33 \mathrm{~W} / \mathrm{m}^{2}$.

A such optimized $\mathrm{ZnO}$ layer is therefore shown to be a viable tool to increase the near-IR absorption of an a-Si:H solar cell. It is important to note that this is achieved without reducing the electrical current generation in the solar cell.

\section{Emissivity for heat-radiation - emission losses}

The second important disadvantage when replacing optimized thermal absorber surfaces by PV modules in a hybrid application is the emissivity behavior of the absorber surface for heat radiation in the far-IR (loss term $B$ in Fig.1.).

The radiation-behavior of an absorber is basically determined by the emission coefficient $\varepsilon$ of the absorber surface for heat-radiation in the far-IR region. A good selective material should have a high value of $\varepsilon(=\alpha)$ in the visible and a low value of $\varepsilon$ in the far-IR $(\lambda>3 \mu \mathrm{m})$. Tab.2. shows measured values of the emissivity for different samples. Interestingly, $\mathrm{SnO}_{2}$ (Asahi U-type) has an $\varepsilon^{-}$ coefficient which is nearly as low as for optimized surfaces for thermal collectors. ZnO has an $\varepsilon$-value which is only one half of that of a glass surface.

\begin{tabular}{|l|l|}
\hline sample & emissivity $\varepsilon$ \\
\hline glass & 0.86 \\
\hline $\mathrm{p}$-i-n (glass on top) & 0.85 \\
\hline $\mathrm{n}$-i-p (ITO on top) & $0.60-0.65$ \\
\hline poly-Si (non-encapsulated) & 0.40 \\
\hline sputtered ZnO & 0.41 \\
\hline $\mathrm{SnO}_{2}$ (Asahi U) & 0.17 \\
\hline commercial selective absorber & $0.10-0.17$ \\
\hline
\end{tabular}

Table 2.: Values for the emissivity $\varepsilon$ for different materials and cell structures.

Highly doped semiconductors show a high reflectivity in the far-IR region [11,12]. Surface texturing further reduces the emissivity in the far-IR due to so-called wavefront discrimination [13] which explains the large difference between the flat $\mathrm{ZnO}$ and the highly textured $\mathrm{SnO}_{2}$. This low emissivity for far-IR, together with high transparency in the range of the visible light, makes TCOs well-suited as a heat-mirror in a tandem absorber surface where the upper layer (TCO) is transparent for the sunlight and has a low emissivity coefficient and the lower layer absorbs the sunlight efficiently [11]. In our case, the TCO $(\mathrm{ZnO})$ additionally is tuned to absorb that part of the sunlight which cannot be absorbed in the amorphous cell.

A layer of $\mathrm{ZnO}$ or $\mathrm{SnO}_{2}$ is generally already incorporated into a standard a-Si:H solar module structure. Intelligent use and a slight adaption of the deposition parameters could substantially contribute to a higher thermal performance of an a-Si: $\mathrm{H}$ based hybrid module without affecting the electrical properties. Such a layer bears the possibility to enhance the thermal performance in a twofold manner: the IR part of the sunlight can made to be absorbed to a larger extent and, additionally, losses due to heat radiation of the module surface can be substantially lowered if the TCO is deposited on top of the structure. Furthermore, such a layer would in the ideal case be completely transparent for light which is converted into electrical energy and would therefore not affect the electrical performance of the module.

It is very important to note that the deposition of such a layer in a solar module is completely compatible with the thin-film deposition process and does not require any additional process step.

\section{The proposed structure}

In the following, we propose a module structure (Fig.6.) which is, in conclusion to the preceeding considerations, very well suited for a hybrid collector and which has due to an enhanced thermal efficiency the potential to result in a 
remarkable increase of the total efficiency of such hybrid collectors operated at high temperatures.

An amorphous cell in the $n$-i-p (substrate) structure can be easily deposited directly on top of a conventional heat exchanger made from copper or steal with an appropriate coverage forming a back reflector and preventing diffusion if needed. This (back-)surface could be textured in order to obtain a maximum light-trapping effect in the cell. The front contact, a thin layer of ITO, is proposed to be replaced by an optimized layer of $\mathrm{ZnO}$. The properties of this top $\mathrm{ZnO}$ should be tailored, as proposed in this paper, such as to obtain the desired properties, i.e. a) absorption of the infrared part of the sunlight converting it into heat, b) high transmissivity for wavelengths contributing to current generation in the cell and c) low emission for heatradiation in the far-IR range. This effect will be largest if the surface of the $\mathrm{ZnO}$ can be made textured. This texture could also help to further increase the light-trapping effect of the solar cell.

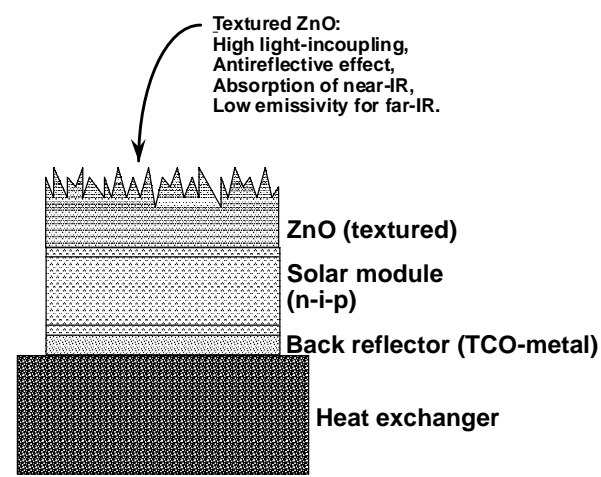

Fig.6.: The structure proposed for an optimized thermal performance of the hybrid collector without affecting the electrical performance of the cell.

A PV module based on an amorphous $n-i-p$ cell together with the proposed increase in IR absorption and reduction of the heat radiation is most suited as absorber layer for a hybrid collector. Its thermal efficiency can come close to values obtained for optimized absorber surfaces if the near-IR absorption (Fig.2.) of the PV module can be increased by a $\mathrm{ZnO}$ layer, and if furthermore the emissivity for heat radiation can be brought down to values comparable to those of optimized selective absorber surfaces. The influence of such a $\mathrm{ZnO}$ layer on the electrical efficiency of the cell has been shown to be negligible.

The proposed structure is therefore able to perform like an optimized thermal collector while at the same time furnishing electrical energy with an efficiency which is determined by the cell performance and not affected by its additional function as a heat absorber.

\section{Conclusions}

Due to economic reasons on the one hand and its low temperature coefficient on the other hand, amorphous silicon is a most promising material for hybrid collectors.

The thermal efficiency of hybrid collectors was found to suffer from incomplete absorption of the sunlight and from losses due to heat-radiation when the module is operated at high temperatures. It was shown that both loss mechanisms can be reduced to a large amount if an optimized TCO layer is incorporated into the module structure.

$\mathrm{ZnO}$ is shown to be very useful in this context due to its low emissivity in the far-IR and due to its possibility to absorb the near-IR part of the sunlight. The deposition of such tailored $\mathrm{ZnO}$ layers is completely compatible with the thin-film deposition process of the solar module.

The optimum structure, as proposed in the paper, consists of an a-Si:H n-i-p solar cell deposited directly on the heat exchanger. The top electrode is formed by a textured $\mathrm{ZnO}$ surface showing the desired properties of low emissivity and absorption of the near-IR.

Such a structure is believed to result in a thermal performance close to that of optimized thermal absorber surfaces and an electrical efficiency which is not affected by the dual use of the solar moduel as absorber surface.

\section{Acknowledgements}

Financial support by Swiss Federal Department of Energy BEW/OFEN under grant 19431 is gratefully acknowledged. R. Platz thanks the Arthur u. Aenne Feindt Stiftung, Hamburg (Germany), the Fondation CharlesEdouard Guillaume, Bienne (Switzerland), and EPRI for funding of his stay at Princeton University.

\section{References}

1 A.V. Shah, R. Platz and H. Keppner, Sol. En. Mat and Sol. Cells 38 (1995) 501.

2 M. Izu, H.C. Ovshinsky, X. Deng, A.J. Krisko, K.L. Narasimhan, R. Crucet, T. Laarman, A. Myatt and S.R. Ovshinsky, Proc. 1st WCPEC (1994) 820.

3 S. Fujikake, K. Tabuchi, A. Takano, T. Wada, S. Saito, H. Sato, T. Yoshida, Y. Ichikawa and H. Sakai, Proc. 25th IEEE PVSC (1996) 1045.

$4 \quad$ M. Götz, H. Keppner, P. Pernet, W. Hotz and A. Shah, MRS Symp. Proc. 426 (1996) 89.

$5 \quad$ K. Bücher, Proc. 13th E-PVSEC (1995) 2097

6 C. Hof, M. Lüdi, M. Götz, D. Fischer and A. Shah, Proc. 25th IEEE PVSC (1996) 1057.

7 A. Ricaud and P. Roubeau, Proc. 25th IEEE PVSC (1996) 1012.

8 B. Lalovic, Z. Kiss and H. Weakliem, Solar Cells 19 (1986-1987) 131

9 J.A. Anna Selvan, H. Keppner and A. Shah, MRS Symp. Proc. 426 (1996) 497.

10 W.W. Molzen, J. Vac. Sci. Technol. 12 (1975) 99.

11 M.M. Koltun, Selective Optical Surfaces for Solar Energy Converters, Allterton Press, Inc., New York 1981.

12 C.A. Escofferey and W. Luft, Solar Energy 4 (1960) 1.

13 H. Tabor, Bull. Res. Counc. Isr. A5 (1956) 119. 